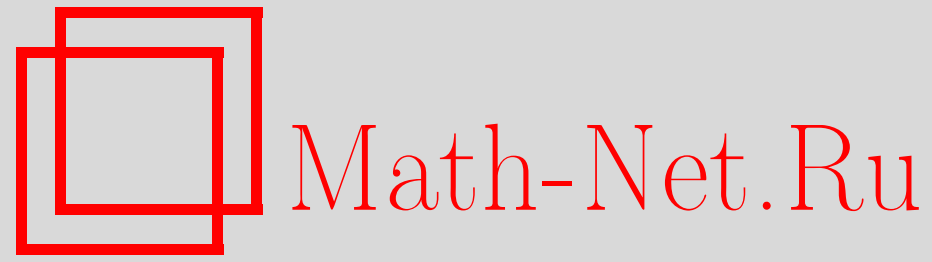

Общероссийский математический портал

В. В. Рыжиков, Четная и нечетная простота динамических систем с инвариантной мерой, Матем. заметки, 1996, том 60, выпуск 3, 470-473

DOI: https://doi.org/10.4213/mzm1854 
Использование Общероссийского математического портала MathNet.Ru подразумевает, что вы прочитали и согласны с пользовательским соглашением

http://www . mathnet.ru/rus/agreement

Параметры загрузки:

IP: 34.227 .88 .159

26 апреля 2023 г., 03:56:25

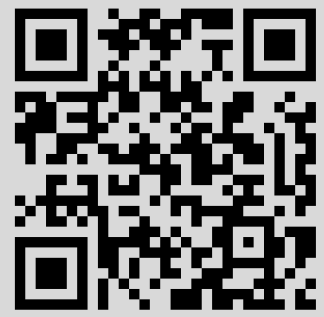




\section{ЧЕТНАЯ И НЕЧЕТНАЯ ПРОСТОТА ДИНАМИЧЕСКИХ СИСТЕМ С ИНВАРИАНТНОЙ МЕРОЙ}

\section{В.В. Рыжиков}

Динамической системой в заметке называется сохраняющее меру $\mu$ действие $\Phi=\left\{T_{g}: g \in G\right\}$ некоторой группы $G$ на пространстве Лебега $(X, \mu), \mu(X)=1$. Говорим, что мера $\nu$, заданная на декартовом кубе $X^{n}$, принадлежит классу $M(n-1, n), n>2$, если проекции $\nu$ на $(n-1)$-мерные грани куба $X^{n}$ равны $\mu^{n-1}$. Говорим, что действие $\Phi$ обладает свойством $S(n-1, n)$ (или говорим, что действие принадлежит классу $S(n-1, n))$, если $\mu^{n}$ - единственная мера класса $M(n-1, n)$, инвариантная относительно действия $\Phi \otimes \Phi \otimes \cdots \otimes \Phi(n$ сомножителей).

Свойства $S(n-1, n)$ рассматривались в работах [1]-[6]. Интерес к ним возник в связи с некоторыми задачами теории джойнингов [3] и исследованием кратного перемешивания (если коммутативная перемешивающая система класса $S(n, n+1)$ перемешивает с кратностью $n-1$, то она перемешивает и с кратностью $n$, см. [5]). Известно (см. [4], [6]), что

i) все (четные) свойства $S(2 p-1,2 p), p>1$, эквивалентны между собой;

ii) свойство $S(3,4)$ влечет за собой любое из свойств $S(2 q, 2 q+1), q=1,2, \ldots$.

Работа выполнена при поддержке Российского фонда фундаментальных исследований, грант № 96-01-00713.

(C) в.в. Рыжиков 1996 
В заметке приводится пример некоммутативного действия $\Psi$, обладающего свойством $S(2 q, 2 q+1)$, но не обладающего свойством $S(2 p-1,2 p)$. В этом случае говорим, что действие $\Psi$ обладает нечетной тензорной простотой. Терминология объясняется следующим. Динамической системе $\Psi$ отвечает группа $\widehat{\Psi}$ унитарных операторов, действующих на пространстве $L_{2}(X, \mu)$. Нечетная тензорная простота означает, что $\widehat{\Psi}$ не допускает нетривиальных марковских сплетений с четными тензорными степенями системы $\widehat{\Psi}$.

Определим действие $\Psi$. Предварительно отметим, что автоморфизмы группы $X=\mathbb{Z}_{2} \times \mathbb{Z}_{2} \times \mathbb{Z}_{2} \times \cdots$ и сдвиги на $X$ сохраняют меру Хаара $\mu$ на $X$. Для перестановки $\sigma$ натурального ряда $\mathbb{N}$ определим автоморфизм $T_{\sigma}$ группы $X$ равенством $T_{\sigma}\left(\left\{x_{i}\right\}\right)=\left\{x_{\sigma(i)}\right\}$. Последовательности $\alpha=\left\{a_{i}\right\} \in X$ сопоставим сдвиг: $S_{\alpha}(x)=x+\alpha$, где '十' обозначает групповую операцию в $X$. В качестве $\Psi$ рассмотрим действие, порожденное всевозможными $T_{\sigma}$ и $S_{\alpha}(x)$.

ТЕОРема 1. При $q>1$ действие $\Psi$ обладает свойствами $S(2 q, 2 q+1)$, но не обладает свойствами $S(2 q+1,2 q+2)$.

ДокАЗАТЕЛЬСтво случая $q=1 . \Psi \notin S(3,4)$, так как мера $\eta$, определенная формулой

$$
\eta(A \times B \times C \times D)=\mu^{3}(\{(a, b, c): a \in A, b \in B, c \in C, a+b+c \in D\}),
$$

отлична от $\mu^{4}$, инвариантна относительно $\Psi \otimes \Psi \otimes \Psi \otimes \Psi$ и принадлежит класcy $M(3,4)$.

Покажем, что $\Psi$ не обладает свойством $S(2,3)$. Пусть мера $\nu \in M(2,3)$ инвариантна относительно $\Psi \otimes \Psi \otimes \Psi$ :

$$
\forall \psi \in \Psi \quad \int \psi \chi_{A} \otimes \psi \chi_{B} \otimes \psi \chi_{C} d \nu=\int \chi_{A} \otimes \chi_{B} \otimes \chi_{C} d \nu
$$

Отметим свойства действия $\widehat{\Psi}: S_{\alpha} \chi_{n}=-\chi_{n}$ при $a_{n}=1 ; S_{\alpha} \chi_{n}=\chi_{n}$ при $a_{n} \neq 1$; $\chi_{\sigma(n)}(x)=\chi_{n}\left(T_{\sigma}(x)\right)$. Если $A \cap B \cap C \neq \varphi$ и $j \in A \cap B \cap C$, то для сдвига $S_{j}$, определенного формулой $\left(S_{j}(x)\right)_{i}=x_{i}+\delta_{i, j}$, имеем

$\int\left(\chi_{A} \otimes \chi_{B} \otimes \chi_{C}\right) d \nu=\int\left(S_{j} \chi_{A} \otimes S_{j} \chi_{B} \otimes S_{j} \chi_{C}\right) d \nu=-\int\left(\chi_{A} \otimes \chi_{B} \otimes \chi_{C}\right) d \nu=0$.

То же самое получим при $j \in A$, когда $j \notin B \cup C$.

Теперь рассмотрим случай, когда $j \notin A$ и $j \in B \cap C$. Найдется перестановка $\sigma$, удовлетворяющая условиям:

a) $\sigma(i)=i$, если $i \in A$;

b) $\sigma^{n}(j) \rightarrow \infty$ при $n \rightarrow \infty$.

Тогда, учитывая инвариантность меры $\nu$ и свойства a), b), получим

$$
\begin{aligned}
& \int\left(\chi_{A} \otimes \chi_{B} \otimes \chi_{C}\right) d \nu=\int\left(T_{\sigma} \chi_{A} \otimes T_{\sigma} \chi_{B} \otimes T_{\sigma} \chi_{C}\right) d \nu \\
& =\int\left(\chi_{A} \otimes T_{\sigma} \chi_{B} \otimes T_{\sigma} \chi_{C}\right) d \nu=\lim _{N \rightarrow \infty} \int\left(\chi_{A} \otimes \sum_{n=1}^{N} \frac{1}{N}\left(T_{\sigma}^{n} \chi_{B} \otimes T_{\sigma}^{n} \chi_{C}\right)\right) d \nu=0 .
\end{aligned}
$$

Последнее равенство вытекает из попарной ортогональности функций

$$
Y_{n}=T_{\sigma}^{n} \chi_{B} \otimes T_{\sigma}^{n} \chi_{C}=\chi_{\sigma^{n}(B)} \otimes \chi_{\sigma^{n}(C)}, \quad n=0,1,2, \ldots
$$


Подводя итог сказанному выше, заключаем, что неравенство $\nu\left(\chi_{A} \otimes \chi_{B} \otimes \chi_{C}\right) \neq 0$ может иметь место только в случае $A=B \sqcup C$ (дизъюнк тное объединение). Однако, по тем же причинам получим $B=C \sqcup A$, следовательно, $A=(C \sqcup A) \sqcup C=A \sqcup C$, что невозможно. Доказано, что $\nu\left(\chi_{A} \otimes \chi_{B} \otimes \chi_{C}\right)=0$ для всех наборов $\chi_{A}, \chi_{B}, \chi_{C}$, следовательно, $\nu=\mu^{3}$. Таким образом, $\Psi \in S(2,3)$. Аналогичные рассуждения показывают, что $\Psi \in S(2 q, 2 q+1)$.

Действие $\Psi$ содержит много поддействий класса $S(2 q, 2 q+1) \backslash S(3,4)$. В частности, к ним относится действие счетной подгруппы, порожденной всеми финитными перестановками $(\operatorname{supp}(\sigma)-$ конечное множество) и финитными сдвигами (сдвигами, для которых последовательности $\alpha=\left\{a_{i}\right\}$ финитны).

Действия с минимальным и простым централизатором. Джсйнингом пары $(\Phi, \Phi)$ назьвается мера $\nu$ на $X \times X$, инвариантная относительно $\Phi \otimes \Phi$ и имеющая проекции на сомножители в $X \times X$, совпадающие с $\mu$.

Следуя терминологии [3], действие $\Phi$ называем 2-простым, если любой эргодический джойнинг $(\Phi, \Phi)$ есть мера $\mu \otimes \mu$ или мера вида $(\operatorname{Id} \otimes R) \Delta$, где $R$ - обратимое преобразование, коммутирующее с $\Phi$, а $\Delta$ - диагональная мера на $X \times X$. Напомним, что $\Delta(A \times B)=\mu(A \cap B)$. Действие $\Phi$ называется $n$ - $п$ ростым, если $\Phi$ является 2-простым и принадлежит классу $\bigcap_{m=3}^{n} S(m-1, m)$.

Если все эргодические джойнинги пары $(\Phi, \Phi)$ есть $\mu \otimes \mu$ и меры вида $\left(\operatorname{Id} \otimes T_{h}\right) \Delta$, где $T_{h}$ входит в действие $\Phi=\left\{T_{g}: g \in G\right\}$, а $h$ лежит в центре группы $G$, говорят, что действие $\Phi$ имеет минимальный марковский чентрализатор. В теории джойнингов принято писать в этом случае $\Phi \in \operatorname{MSJ}(2)$. Обозначим $\operatorname{MSJ}(n)=$ $\operatorname{MSJ}(2) \cap \bigcap_{m=3}^{n} S(m-1, m)$.

Для $\mathbb{Z}$-действий выполнено $\mathrm{MSJ}(3)=\mathrm{MSJ}(4)$, для них имеет место также более общий факт: 3 -простота влечет за собой $n$-простоту (см. [1], [4], [6]). Однако, для некоммутативных динамических систем аналогичное утверждение, как будет показано, вообще говоря, неверно.

ТЕОРема 2. 1) Действие $\Psi$, порожсденное всеми автоморфизмами әруппь $Y=\mathbb{Z}_{3} \times \mathbb{Z}_{3} \times \mathbb{Z}_{3} \times \cdots$ и всеми групповыми сдвигами на $Y$, принадлежст классу MSJ(2), но не принадлежст классу MSJ(3). Действие $\Phi$, порожсденное всеми автоморфизмами группь $X=\mathbb{Z}_{2} \times \mathbb{Z}_{2} \times \mathbb{Z}_{2} \times \cdots$ и всеми сдвигами на әруппе $X$, принадлежит классу MSJ(3) \MSJ(4). Эргодическими джойнингами как $(\Phi, \Phi)$ так $u(\Psi, \Psi)$ являются только меры $\Delta u \mu \otimes \mu$.

2) Классу MSJ(3)\MSJ(4) принадлежст действие $\Phi^{\prime}$, порожсденное (бернуллиевским) автоморфизмом $T$ на $\cdots \times \mathbb{Z}_{2} \times \mathbb{Z}_{2} \times \mathbb{Z}_{2} \times \cdots$ и инволюииями $R, S$, определенными следуюшим образом:

$$
\begin{gathered}
T(x)_{i}=x_{i+1}, \\
S\left(\ldots, x_{-2}, x_{-1}, x_{0}, x_{1}, x_{2}, \ldots\right)=\left(\ldots, x_{-2}, x_{-1}, x_{1}, x_{0}, x_{2}, \ldots\right), \\
R\left(\ldots, x_{-2}, x_{-1}, x_{0}, x_{1}, x_{2}, \ldots\right)=\left(\ldots, x_{-2}, x_{-1}, x_{0}, x_{1}+x_{0}, x_{2}, \ldots\right) .
\end{gathered}
$$

СледСтвИЕ. Простота порядка 2, вообще говоря, не влечет простоту порядка 3. Простота порядка 3, вообще говоря, не влечет простоту порядка 4 .

Почти простые действия. Марковским оператором $Q: L_{2}(X, \mu) \rightarrow$ $L_{2}(X, \mu)$ назьвается оператор, который переводит неотрицательные функции в неотрицательные, а постоянные функции - в себя, причем сопряженный оператор $Q^{*}$ также обладает этими свойствами. Будем говорить, что действие $\Psi$ имеет почти простой марковский централизатор $\mathrm{SC}(\Psi)$, если для некоторого $a>0$ найдется конечный набор марковских операторов $\left\{Q_{i}\right\}, Q_{i} \in \mathrm{SC}(\Psi)$, удовлетворяющих условиям:

1) $Q_{i}^{*} Q_{i}>a I, Q_{i} Q_{i}^{*}>a I$ 
2) каждый марковский оператор $J \neq \Theta$, коммутирующий с действием $\Psi$, имеет вид

$$
J=a \Theta+\sum_{i} a_{i} Q_{i} \int_{C(\Psi)} R d \lambda_{i}(R), \quad a+\sum_{i} a_{i}=1,
$$

где $\Theta$ обозначает оператор ортопроекции напространство постоянных функций, $R$ - автоморфизмы из группового централизатора $C(\Psi)$ действия $\Psi$, а $\lambda_{i}$ есть некоторые вероятностные меры на $C(\Psi),(I$ - тождественный оператор).

ТЕОРема 3. Если иентрализатор почти простого действия содерхит слабо перемешивающий, но не перемешиваюший автоморфизм, то это действие принадлежст классу $S(3,4)$. Для почти простого $\mathbb{Z}^{n}$-действия для любого $m \geqslant 1$ свойство $S(2 m, 2 m+1)$ әквивалентно свойству $S(3,4)$.

Косые произведения, сохраняющие тензорную простоту. Известно, что перемешивающие групповые расширения сохраняют ряд свойств динамической системы, среди которых - свойство тензорной простоты. Как показано ниже, имеются другие естественные типы расширений, сохраняющие свойства $S(n-1, n)$.

Tеорема 4. Пусть $R: X \times X \rightarrow X \times X-$ косое произведение, заданное равенством $R(x, y)=\left(S(x), T_{s}(x)(y)\right)$, әде $T_{t}-$ перемешиваюший поток и $\int s(x) d \mu(x)=0$. Пусть $R$ сохраняет меру $\nu=\mu \otimes \mu$ и обладает свойством перемешивания.

Тогда, а) если $S$ из класса $S(p-1, p)$, то косое произведение $R$ такжсе принадлежсит классу $S(p-1, p)$, б) если $S$ перемешивает с кратностью $k$, то $R$ также перемешивает с кратностью $k$, m.е. при $n_{1}, n_{2}, \ldots, n_{k} \rightarrow \infty$ $u\left|n_{i}-n_{j}\right| \rightarrow \infty(1 \leqslant i<j \leqslant k)$, выполнено

$$
\nu\left(B_{0} \mid R^{n_{1}} B_{1} \cap R^{n_{2}} B_{2} \cap \cdots \cap R^{n_{k}} B_{k}\right) \rightarrow \nu\left(B_{0}\right)
$$

для любих множеств $B_{0}, B_{1}, B_{2}, \ldots, B_{k}$ полохительной $\nu$-меры.

Автор обязан А. М. Стёпину за обсуждение заметки и ценные замечания.

Московский государственный университет

Поступило им. М.В. Ломоносова

29.03 .96

E-mail: vryz@nw.math.msu.su

\section{СПИСОК ЦИТИРОВАННОЙ ЛИТЕРАТУРЫ}

1. Glasner E., Host B., Rudolph D. // Israel J. Math. 1992. V. 78. P. 131-142. 2. Host B. // Israel J. Math. 1991. V. 76. P. 289-298. 3. del Junco A., Rudolph D. // Ergod. Th. Dynam. Sys. 1987. V. 7. P. 531-557. 4. King J. // Israel J. Math. 1992. V. 80. P. 65-86. 5. Рыжиков В. В. // УМН. 1989. Т. 44. №1. C. 205-206. 6. Ryzhikov V. V. // J. Dynamical Control Sys. 1996. V. 2. №1. P. 1-19. 